\title{
Quasi-rational fusion products
}

\author{
Werner Nahm
}

Bonn University

abstract: Fusion is defined for arbitrary lowest weight representations of $W$-algebras, without assuming rationality. Explicit algorithms are given. A category of quasirational representations is defined and shown to be stable under fusion. Conjecturally, it may coincide with the category of representations of finite quantum dimensions.

\section{Introduction}

In conformal quantum field theories in two dimensions the role of tensor products of Lie algebra representations is taken over by the fusion of lowest weight representations of $W$ algebras. This is one of the central concepts of conformal theory, and has been much studied from various mathematical points of view [FF 88, KL 93, FFK 89,90, G 93]. None of the extant approaches, however, is general enough for all purposes and applies, e.g., to the Virasoro algebra for non-minimal values of the central charge. Equally important, a lot of work still has to be done to develop good fusion algorithms for general $W$-algebras. So far, the most important partial results concern rational theories on the one hand and unitary theories on the other. For rational theories, fusion of lowest weight representations is certainly calculable by existing methods, at least in principle. In particular, the characters of all representations can be calulcated from a finite number of terms [MMS 88]. Once they are known, fusion multiplicities are given by the Verlinde formula [V 88]. Apart from questions of computer memory, this is sufficient for many purposes, though problems like the construction of the corresponding braid group representations require additional information.

Non-rational conformal theories are as important for physics as the rational ones. For example, the phenomenologically interesting Calabi-Yau theories depend on several continuous parameters. To yield a rational theory, these must take special values, which are not necessarily the ones required by phenomenology. Not much is known about fusion in non-rational theories, though it may be well behaved. Consider, e.g., the simple case of free bosons on a torus. Here the torus metric acts as free parameter. For generic metrics, the theories are not rational, but the fusion product still is simple. In fact, it just is given by the addition of the abelian charges.

Perhaps fusion always has the quasi-rational features seen for the free bosons. It will be important to investigate Calabi-Yau spaces from this point of view, but the tools for this study have yet to be developed. As a first step, we need a rigorous and convenient definition of the fusion product for generic theories, and better algorithms for its evaluation.

Unitary quantum field theories have been treated with the methods of operator algebra [FRS 89]. Some results reach beyond what has been established for conformal theories, but the differences in the axioms make the comparison somewhat difficult. In particular, unitarity plays no special role in the conformal context, but so far has been crucial for the application of operator algebras. On the other hand, conformal invariance itself is not essential for the latter approach.

In the following, first a concise definition of fusion will be given. If follows the established ideas, but puts more emphasis on generality and algorithms. A suitably defined category 
of quasi-rational representations will be shown to be stable under fusion. These representations have finite dimensional special subspaces, whose dimensions behave submultiplicative under fusion. In particular, this yields finite upper bounds on the irreducible representations which occur in the fusion product. Related finite upper bounds are given for the fusion of quasirational representations with arbitrary lowest weight representations.

The quasirational category is contained in the category of representations with finite quantum dimension, for which operator algebra also suggests stability under fusion. I conjecture that both categories are equal and sufficiently large to treat all conformal theories. Note, however, that theories with charges at infinity will not be considered, since they do not fulfil all axioms of conformal field theory. For many fusion calculations they are essential as auxiliary constructions, but not for the ones in this paper. In particular, the fields of our $W$-algebras all will have positive dimensions.

\section{A definition of the fusion product}

For a given $W$-algebra $\mathcal{A}$ consider the category $\mathcal{V}$ of those lowest weight modules which involve only finitely many irreducible representations and finitely many linearly independent vectors of any bounded degree. By the appropriate version of the CPT theorem, $\mathcal{V}$ admits a duality functor which maps every representation space $V$ to its dual $V^{v}$. Let $\mathcal{A}_{0}$ be the zero mode algebra of $\mathcal{A}$ and $\mathcal{A}_{ \pm}$the subspaces of positive and negative modes of $\mathcal{A}$. For $V \in \mathcal{V}$, let $V^{l}$ the subspace of $V$ which is annihilated by $\mathcal{A}_{-}$. Every representation $V \in \mathcal{V}$ is determined by the action of $\mathcal{A}_{0}$ on the lowest weight spaces $V^{l}$ and $\left(V^{v}\right)^{l}$. The zero mode algebra representation on $V^{l}$ inherits the Lie bracket of the $W$-algebra and an expression of the zero modes of normal ordered products in terms of the zero modes of the component fields. The natural pairing between $\mathcal{A}_{+} \otimes V^{l}$ and $\mathcal{A}_{+} \otimes\left(V^{v}\right)^{l}$ is the Shapovalov form.

Most representations encountered in conformal theory are completely reducible, which implies $\left(V^{v}\right)^{l}=\left(V^{l}\right)^{v}$, such that a separate consideration of $V^{v}$ is not necessary. In this case, $V$ is given by $\mathcal{A}_{+} V^{l}$ modulo its maximal submodule which does not intersect $V^{l}$. More general representations may be treated in a similar way.

Consider some conformal theory $\mathcal{T}^{\alpha}$ with $W$-algebra $\mathcal{A}$ and let $V_{1}, V_{2} \in \mathcal{V}$. Consider a Riemann sphere with three punctures $z_{1}, z_{2}, z_{3}$. When the $V_{i}$ are attached to $z_{i}$ for $i=1,2$, they will produce some representation $V_{3}^{\alpha}$ attached to $z_{3}$. The fusion product $V_{12}$ of $V_{1}$ and $V_{2}$ should yield a universal module $V_{3}$ which contains all possible $V_{3}^{\alpha}$ as factor modules and is not unnecessarily large. A subcategory $\mathcal{V}^{\prime}$ of $\mathcal{V}$ will be called stable under fusion, if $V_{1}, V_{2} \in \mathcal{V}^{\prime}$ implies $V_{12} \in \mathcal{V}^{\prime}$.

By linearity, $V_{12}$ should be a suitable completion of $V_{1}\left(z_{1}\right) \otimes V_{2}\left(z_{2}\right)$. As a vector space, the latter space is just $V_{1} \otimes V_{2}$, but the action of the $W$-algebra depends on $z_{1}, z_{2}$, which explains the notation. To define this action, let $C$ be a small circle around $z_{3}$. For holomorphic fields $\phi$ of $\mathcal{A}$, the vector $\phi(z) v, v \in V_{1}\left(z_{1}\right) \otimes V_{2}\left(z_{2}\right)$ must depend holomorphically on $z$, except for possible poles at $z_{1}, z_{2}$. Thus for 1 -forms $d \omega$ which are holomorphic away from $z_{1}, z_{2}$, we must have

$$
\oint_{C} \phi(z) d \omega\left(v_{1}\left(z_{1}\right) \otimes v_{2}\left(z_{2}\right)\right)=\left(\oint_{C_{1}} \phi(z) v_{1}\left(z_{1}\right) d \omega\right) \otimes v_{2}\left(z_{2}\right)+v_{1}\left(z_{1}\right) \otimes \oint_{C_{2}} \phi(z) v_{2}\left(z_{2}\right) d \omega
$$


where $C_{1}, C_{2}$ are small circles around $z_{1}, z_{2}$. The integrals along them are given by the $W$-algebra modules $V_{1}, V_{2}$.

This fact defines a natural action of the $W$-algebra on the tensor product itself. The commutativity and associativity properties of the tensor product will induce corresponding properties of the fusion product defined below. The $W$-algebra action depends on $z_{1}, z_{2}$, but different choices of the $z_{i}$ are related by rational diffeomorphisms and lead to isomorphic modules. When no ambiguity arises, we omit the explicit notation of the $z_{i}$ dependence. Using such a rational transformation of the Riemann sphere, we put $z_{3}=\infty$.

A more subtle question concerns the topology of the product space. Consider for example vacuum representation $V_{1}, V_{2}$ and the Virasoro field $L$. We have

$$
\oint_{C} L(z)\left(z-z_{1}\right)^{-1} d z=\sum_{n=1}^{\infty}\left(z_{1}-z_{2}\right)^{n-1} \oint_{C} L(z)\left(z-z_{2}\right)^{-n} d z
$$

Evaluating this relation on the product of two vacuum states $v_{0}$, we obtain

$$
\left(L_{2} v_{0}\right) \otimes v_{0}=\sum_{n=2}^{\infty}\left(z_{1}-z_{2}\right)^{n-2} v_{0} \otimes\left(L_{n} v_{0}\right)
$$

which certainly is not an obvious limit in the ordinary tensor product. Moreover, the lowest weight vectors in $V_{12}$ will be given by similar limits.

More generally, the vectors $\oint_{C} \phi(z) d \omega v, v \in V_{1} \otimes V_{2}$ must depend continuously on $d \omega$. For example, we want to write

$$
\oint_{C} \phi(z) f(z) d z v=\sum_{n=n_{0}}^{\infty} a_{n} \oint_{C} \phi(z) z^{-n} d z v
$$

where $f(z)=\sum a_{n} z^{-n}$ is the Laurent expansion of $f(z)$ around $z_{3}$. The importance of the topology becomes obvious in the proof of the following basic fact.

Fusion with the basic representation leaves lowest weight representations invariant.

Proof: Let $V_{1}$ be the basic representation and $\phi \in V_{1}$. For $v \in V_{2}$ we have

$$
\phi\left(z_{1}\right) \otimes v=\oint_{C} \phi(z) d \omega(1 \otimes v)-1 \otimes \oint_{C_{2}} \phi(z) d \omega v
$$

where $d \omega=\left(2 \pi i\left(z-z_{1}\right)\right)^{-1} d z$. Now on $C$ we can approximate $d \omega$ by differentials $d \omega_{N}$ which are regular at $z_{1}$, such that

$$
\oint_{C} \phi(z) d \omega_{N}(1 \otimes v)=1 \otimes \oint_{C_{2}} \phi(z) d \omega_{N} v=1 \otimes \oint_{C} \phi(z) d \omega_{N} v
$$

Passing to the limit $d \omega$ we find

$$
\phi\left(z_{1}\right) \otimes v=1 \otimes \phi\left(z_{1}\right) v
$$


where $\phi\left(z_{1}\right) v$ is a vector in $V_{2}$. q.e.d.

We circumvent the topological problems by considering the dual modules. The lowest weight vectors in $V_{12}^{v}$ are contained in $\left(V_{1} \otimes V_{2}\right)^{v}$. Let $V_{12}^{A}$ be the subspace of $V_{1} \otimes V_{2}$ spanned by all vectors of the form $\phi_{n} v, n>0, v \in V_{1} \otimes V_{2}$. Let $V_{3}^{l}$ be the subspace of $\left(V_{1} \otimes V_{2}\right)^{v}$ which annihilates $V_{12}^{A}$. Since the zero mode algebra $\mathcal{A}_{0}$ leaves $V_{12}^{A}$ invariant, $V_{3}^{l}$ carries a representation of $\mathcal{A}_{0}$. When $V_{3}^{l}$ is finite dimensional, it induces a lowest weight representation $V_{3} \in \mathcal{V}$. Thus we can define the fusion product by $V_{12}=V_{3}^{v}$. When $V_{3}^{l}$ is infinite, its finite dimensional subrepresentations induce a net of representations $V_{\alpha} \in \mathcal{V}$ which does not have a maximal element. In this case, we identify the fusion product with the inverse limit of the $V_{\alpha}$.

We now will consider a condition which guarantees that $V_{3}^{l}$ is finite dimensional. This condition can be checked explicitly and seems to be true for all conformal theories for which $\mathcal{A}$ is known. It may well be a property of all conformal theories together.

\section{Quasi-rational representations}

Let $\phi$ be a $W$-algebra field of dimension $h(\phi)$. When $d \omega$ vanishes at infinity, then $\oint_{C} \phi(z) d \omega$ belongs to the completion of the subspace $\mathcal{A}_{++}$of $\mathcal{A}$ which is spanned by the $\phi_{n}$ with $n \geq h(\phi)$. In particular, it annihilates any lowest weight state in the dual representation. Thus let us define $V_{12}^{a}$ as the span of vectors of the form $\oint_{C} \phi(z) f(z) d z v, v \in V_{1}\left(z_{1}\right) \otimes$ $V_{2}\left(z_{2}\right)$, where the meromorphic function $f(z)$ vanishes at infinity. Obviously, this space is annihilated by $V_{3}^{l}$.

Let $V_{i}^{a}=\mathcal{A}_{++} V_{i}$. Define special graded subspaces $V_{i}^{s}, V_{12}^{s}$ such that $V_{i}^{s} \oplus V_{i}^{a}$ is dense in $V_{i}$, and analogously for $V_{12}$. For some general considerations one might prefer to consider the factor space $V / V^{a}$ instead, but in concrete calculations one often has to choose some special subspace $V^{s}$, such that the subspace terminology is more convenient. Notice, however, than $\operatorname{dim}\left(V^{s}\right)=\operatorname{dim}\left(V / V^{a}\right)$ does not depend on any choice.

Now we can state our first major result:

Special subspaces are submultiplicative, in other words $V_{1}^{s} \otimes V_{2}^{s}$ contains a special subspace $V_{12}^{s}$.

Proof: We prove this fact by an explicit reduction algorithm which represents any vector in $V_{1} \otimes V_{2}$ as a sum of vectors in $V_{1}^{s} \otimes V_{2}^{s}$ and $V_{12}^{a}$. It is sufficient to consider the dense subspace spanned by vectors $v_{1} \otimes v_{2}$, such that the $v_{i} \in V_{i}$ have homogeneous degrees $\operatorname{deg}\left(v_{i}\right)$. The reduction algorithm decreases the sum $\operatorname{deg}\left(v_{1}\right)+\operatorname{deg}\left(v_{2}\right)$. Since the $V_{i}$ are lowest weight representations, it terminates. A special $V_{12}^{s} \subset V_{1}^{s} \otimes V_{2}^{s}$ then can be chosen as complement of the intersection with $V_{12}^{a}$.

We first decompose every vector into components in $V_{1}^{a} \otimes V_{2}$ and $V_{1}^{s} \otimes V_{2}$. The space $V_{1}^{a}$ is spanned by vectors of the form $v_{1}=\phi_{n+h(\phi)} v_{1}^{\prime}, n \geq 0$, such that $\operatorname{deg}\left(v_{1}^{\prime}\right)=\operatorname{deg}\left(v_{1}\right)-$ $n-h(\phi)$. We have

$$
v_{1}=\oint_{C_{1}}\left(z-z_{1}\right)^{-n-1} \phi(z) v_{1}^{\prime} d z / 2 \pi i
$$

We replace the integral along $C_{1}$ by integrals along $C$ and $C_{2}$. The first one gives a 
contribution to $V_{12}^{a}$, such that we obtain the reduction formula

$$
\phi_{n+h(\phi)} v_{1}^{\prime} \otimes v_{2} \equiv-\oint_{C_{2}}\left(z-z_{1}\right)^{-n-1} \phi(z) v_{2} d z / 2 \pi i \quad \text { modulo } \quad V_{12}^{a}
$$

Since the differential is holomorphic inside $C_{2}$, the right hand side is easy to evaluate and yields

$$
v_{1} \otimes v_{2} \equiv v_{1}^{\prime} \otimes \sum_{k=1}^{\infty}(-)^{n}\left(\begin{array}{c}
n+k \\
k-1
\end{array}\right)\left(z_{1}-z_{2}\right)^{-n-k} \phi_{h(\phi)-k} v_{2} .
$$

Thus it is a sum of vectors of degrees $\operatorname{deg}\left(v_{2}\right)+h(\phi)-k+\operatorname{deg}\left(v_{1}^{\prime}\right)$, all less than $\operatorname{deg}\left(v_{1}\right)+$ $\operatorname{deg}\left(v_{2}\right)$. Vectors in $V_{1}^{s} \otimes V_{2}$ are decomposed into components in $V_{1}^{s} \otimes V_{2}^{s}$ and $V_{1}^{s} \otimes V_{2}^{a}$. The latter are reduced analogously. q.e.d.

In particular,

$$
\operatorname{dim}\left(V_{12}^{s}\right) \leq \operatorname{dim}\left(V_{1}^{s}\right) \operatorname{dim}\left(V_{2}^{s}\right),
$$

such that the subcategory $\mathcal{V}^{q}$ given by the modules $V \in \mathcal{V}$ with finite $\operatorname{dim}\left(V^{s}\right)$ is stable under fusion. The modules of this subcategory will be called quasirational. If $\mathcal{V}^{q}=\mathcal{V}$, then $\mathcal{A}$ itself will be called quasirational. The vacuum representation $V_{0}$ always belongs to $\mathcal{V}^{q}$, since $V_{0}^{s}$ is just the one dimensional ground state, in agreement with the result that $V_{0}$ is the identity of the fusion product.

So far, no conformal theories without quasirational $W$-algebra has been shown to exist, though for generic Calabi-Yau theories the question remains open.

If some $V$ belongs to $\mathcal{V}^{q}$, it is possible to give a constructive proof of this fact. Consider a complete set of simple fields $\phi^{i}$ which generates all others by linear combinations, derivatives, and normal ordered products.

Proposition: Let $V^{\prime}$ be invariant modulo $V^{a}$ under the action of the Fourier components $\phi_{n}^{i}, n<h\left(\phi^{i}\right)$. Then $V^{\prime}$ contains a special subspace.

We have to check that $V^{\prime}+V^{a}$ is dense in $V$. Since $V^{\prime}$ generates $V$, the latter space is spanned by vectors $v \in \mathcal{V}$ which are given by the action of monomials in field components $\phi_{n}^{i}$ on graded vectors $v^{\prime} \in V^{\prime}$. Any such vector will be reduced to a sum of vectors in $V^{\prime}$ and $V^{a}$ by the following algorithm. Commute all field components in $\mathcal{A}_{++}$to the left and collect vectors in $V^{a}$. For the remaining monomials, act with the rightmost field component on $v^{\prime}$ and rewrite the result as a sum of vectors in $V^{\prime}$ and $V^{a}$, which is possible by assumption. Then iterate the procedure. To show that this algorithm terminates, let the complexity of such a vector $v$ be given by the sum of the conformal dimensions $h\left(\phi^{i}\right)$ of all monomial factors plus $\operatorname{deg}\left(v^{\prime}\right)$. Taking commutators lowers complexity, such that the first reduction step works. Subsequent acting with $\phi_{n}^{i}$ on $v^{\prime}$ yields a linear combination of a vector $v^{\prime \prime} \in V^{\prime}$, and vectors of the form $\phi_{m}^{j} w$. The former has degree $n+\operatorname{deg}(v)<h\left(\phi_{i}\right)+\operatorname{deg}\left(v^{\prime}\right)$, the latter have degrees $h\left(\phi^{j}\right)+\operatorname{deg}(w) \leq n+\operatorname{deg}(v)$. Thus in each step the complexity decreases, and the algorithm terminates after reducing $v$ to a finite sum in $V^{\prime}+V^{a}$.

Conversely, if $V^{\prime}$ contains a special subspace, then $V^{\prime}+V^{a}$ is dense in $V$, such that $V^{\prime}$ fulfils the condition of the proposition. Now for sufficiently large $N$ any finite dimensional $V^{s}$ is contained in the subspace $V^{N}$ spanned by the vectors of degree less than $N$. When 
the representation is quasirational, then $V^{N}$ will satisfy the condition of the proposition for sufficiently large $N$. Since these spaces are finite dimensional, quasirational representations can be recognized by finite computations, whenever there is a finite complete set of simple fields $\phi^{i}$. The $W$-algebras of conformal theories all seem to have this property.

On the other hand, one still has to find general procedures to prove that some representation is not quasirational. Explicit expressions for the Shapovalov form are certainly sufficient to do this, but in general cases they may be difficult to obtain.

The intersection $V_{12}^{\sigma}$ of $V_{1}^{s} \otimes V_{2}^{s}$ with $V_{12}^{a}$ will be called the spurious subspace. The action of the $W$-algebra gives an injective map from $\mathcal{A}_{++} \otimes V_{12}^{s}$ to $\left(\mathcal{A}_{++} \otimes V_{1}^{s}\right) \otimes\left(\mathcal{A}_{++} \otimes V_{2}^{s}\right)$. When the natural maps from $\mathcal{A}_{++} \otimes V_{i}^{s}$ to $V_{i} / V_{i}^{s}$ are injective, in another terminology when there are no null field relations in the $V_{i}$, then the spurious subspace vanishes and the calculation of the fusion products is straightforward.

More generally, consider the spaces of null field relations as modules of the algebra $\mathcal{A}^{++}$ generated by $\mathcal{A}_{++}$and find bases (more precisely, generating sets, since the module is not free). When $R_{1}, R_{2}$ are such relation bases for the representations $V_{1}, V_{2}$, one has to reduce $R_{1} \otimes V_{2}^{s}$ and $V_{1}^{s} \otimes R_{2}$ to a vector space of relations in $\left(V_{1}^{s} \otimes V_{2}^{s}\right)+V_{12}^{a}$ according to the algorithm described above. That relation space produces $V_{12}^{\sigma}$.

Relations are given by the kernel of the Shapovalov form, but without knowledge of this form it may be difficult to prove that a generating set of relations is complete. Nevertheless, for a given set of relations the previous calculations yield lower bounds on $V_{12}^{\sigma}$. On the other hand, braid group representation theory yields upper bounds. When the bounds coincide, the spurious subspace is known and the fusion product is completely determined by $V_{12}^{s}=\left(V_{1}^{s} \otimes V_{2}^{s}\right) / V_{12}^{\sigma}$. In concrete cases, this procedure seems to work quite well, but it still is an open problem to devise an algorithm which always will do the job.

Operator algebra results suggest that the category $\mathcal{V}^{f}$ of representations with finite quantum dimensions also is stable under fusion, since quantum dimensions behave multiplicatively under fusion. Since they are $\geq 1$ and additive under direct sums, all representations in $\mathcal{V}^{f}$ involve only a finite number of irreducible ones, such that one should have $\mathcal{V}^{f} \subset \mathcal{V}$. To make general use of operator algebra arguments, however, one should remove the unitary constraint and prove that the quantum dimensions of conformal theory coincide with those of the operator algebraic approach.

It also would be nice to prove $\mathcal{V}^{f}=\mathcal{V}^{q}$. I don't know any counterexample, but only $\mathcal{V}^{q} \subset \mathcal{V}^{f}$ is obvious on first sight. Indeed, $V^{s}+\mathcal{A}_{++} V^{s}$ is dense in $V$. On the other hand, the vacuum representation is essentially given by $\mathcal{A}_{++}$itself. Thus the quantum dimension of the $V$ is $\leq \operatorname{dim}\left(V_{i}^{s}\right)$. Equality holds, when there are no null field relations in the $V_{i}$. In the latter case, the multiplicative behaviour of the quantum dimensions is obvious.

Let us consider explicit calculations of the fusion product. For simplicity, we only consider irreducible representations. Let $V_{2}^{A}$ be the space of vectors of the form $\phi_{n} v, n>0$, such that $V_{2}^{l} \oplus V_{2}^{A}$ is dense in $V_{2}$.

With a slight complication of the previous arguments we prove

$$
V_{1} \otimes V_{2} \equiv V_{1}^{s} \otimes V_{2}^{l} \quad \operatorname{modulo} \quad V_{12}^{A}
$$

using an explicit reduction algorithm from $\mathcal{A}_{++} V_{1}^{s} \otimes \mathcal{A}_{+} V_{2}^{l}$ to $V_{1}^{s} \otimes V_{2}^{l}$. 
Proof: Let us put $z_{2}=0$. For homogeneous vector $v_{1} \otimes v_{2}$ we reduce $\operatorname{deg}\left(v_{2}\right)$, or $\operatorname{deg}\left(v_{1}\right)$ for fixed $\operatorname{deg}\left(v_{2}\right)$. We first reduce to $V_{1}^{s} \otimes V_{2}$. Thus we consider $v_{1}=\phi_{h+n} v_{1}^{\prime}, h=h(\phi)$, $n \geq 0$. We have

$$
\begin{aligned}
& v_{1} \otimes v_{2}=\oint_{C_{1}}\left(z-z_{1}\right)^{-n-1} \phi(z) d z v_{1}^{\prime} / 2 \pi i \otimes v_{2} \\
& \equiv v_{1}^{\prime} \otimes \oint_{C_{2}}\left(z-z_{1}\right)^{-n-1} \phi(z) d z v_{2} / 2 \pi i .
\end{aligned}
$$

Now we expand $\left(z-z_{1}\right)^{-n-1}$ in a Taylor series around 0 . This yields

$$
\begin{aligned}
& v_{1} \otimes v_{2} \equiv \\
& (-)^{n+1} \sum_{k=0}^{h-2}\left(\begin{array}{c}
n+k \\
n
\end{array}\right) z_{1}^{-n-k-1} \oint_{C_{1}} z^{k} \phi(z) d z v_{1}^{\prime} / 2 \pi i \otimes v_{2} \\
& +(-)^{n} v_{1}^{\prime} \otimes \sum_{k=h-1}^{\infty}\left(\begin{array}{c}
n+k \\
n
\end{array}\right) z_{1}^{-n-k-1} \oint_{C_{2}} z^{k} \phi(z) d z v_{2} / 2 \pi i .
\end{aligned}
$$

In the first term on the r.h.s. we expand $z^{k}$ as a polynomial in $z-z_{1}$. Altogether this yields

$$
\begin{aligned}
& v_{1} \otimes v_{2} \equiv \\
& (-)^{n+1} \sum_{k=0}^{h-2} \sum_{l=0}^{h-2-k} \frac{(n+k+l) !}{n ! k ! l !} z_{1}^{-n-k-1} \phi_{h-k-1} v_{1}^{\prime} \otimes v_{2} \\
& +(-)^{n+1} v_{1}^{\prime} \otimes \sum_{k=h-1}^{\infty}\left(\begin{array}{c}
n+k \\
n
\end{array}\right) z_{1}^{-n-k-1} \phi_{h-k-1} v_{2} .
\end{aligned}
$$

The terms on the right hand side of this reduction formula have either lower degree in $V_{2}$ or fixed degree in $V_{2}$ and lower degree in $V_{1}$. Iterating the procedure we end up with $v_{1} \in V_{1}^{s}$, and we only have to consider $v_{1} \otimes v_{2}$ with $v_{2}=\phi_{n} v_{2}^{\prime}, n>0$. As above

$$
v_{1} \otimes v_{2} \equiv \sum_{k=1}^{\infty}(-)^{k}\left(\begin{array}{c}
n-h+k \\
k-1
\end{array}\right) z_{1}^{-n+h-k} \phi_{h-k} v_{1} \otimes v_{2}^{\prime} .
$$

Since $v_{2}^{\prime}$ has lower degree than $v_{2}$, the algorithm will terminate. q.e.d.

As before, one obtains the corollary

$$
\operatorname{dim}\left(V_{12}^{l}\right) \leq \operatorname{dim}\left(V_{1}^{s}\right) \operatorname{dim}\left(V_{2}^{l}\right) .
$$

In particular, this implies that $V_{1} \in \mathcal{V}^{q}, V_{2} \in \mathcal{V}$ implies $V_{12} \in \mathcal{V}$. In other words, the category of general lowest weight representations remains stable under fusion with quasirational representations.

The formula

$$
\phi_{0}\left(v_{1}(z) \otimes v_{2}\right)=\sum_{k=0}^{h-1}\left(\begin{array}{c}
h-1 \\
k
\end{array}\right) z^{k}\left(\phi_{k} v_{1}\right)(z) \otimes v_{2}+v_{1}(z) \otimes \phi_{0} v_{2}
$$


yields the explicit representation of $\mathcal{A}_{0}$ on $V_{1}^{s} \otimes V_{2}^{l}$ modulo its intersection with the spurious subspace, once the right hand sight is reduced as in the previous calculation. More precisely, one finds an explicit map from $\mathcal{A}_{0} \otimes V_{1}^{s} \otimes V_{2}^{l}$ to $V_{1}^{s} \otimes V_{2}^{l}$ itself, once the projections of the vectors $\phi_{n} v, n<h(\phi), v \in V_{1}^{s}$ to $V_{1}^{s}$ and $V_{2}^{a}$ have been calculated. All of this is given by finite computations, but due to the possibility of spurious vectors only gives upper bounds on the fusion multiplicities.

Some spurious representations can be thrown out quickly. If $R$ is a normal ordering relations in $\mathcal{A}_{0}$, then the image of $R \otimes V_{1}^{s} \otimes V_{2}^{l}$ under the reduction procedure is spurious. Moreover, the commutativity of the fusion product allows to remove all irreducible subrepresentations of $\mathcal{A}_{0}$ on $V_{1}^{s} \otimes V_{2}^{l}$ which do not occur in $V_{1}^{l} \otimes V_{2}^{s}$. Still, the example of the Kac-Moody algebras shows that this is not sufficient. For them, $V^{s}=V^{l}$, such that our explicit algorithm just reduces to the calculation of the ordinary tensor product of the underlying finite dimensional Lie algebras and does not yet detect the spurious subspace, which requires explicit consideration of null field relations.

\section{Examples}

Let us illustrate the algorithm for some representations of the Virasoro algebra with $c=$ $1-6(p-q)^{2} / p q$. Let $h(r, s)=\left((p r-q s)^{2}-(p-q)^{2}\right) / 4 p q$, wit $p, q, r, s$ not necessarily integral. Let $V_{r, s}$ be the irreducible module with lowest weight $h(r, s)$. For integral $r, s$ the representations are minimal or degenerate and in any case quasirational, whereas the other representations have infinite quantum dimensions.

In $V_{2,1}$ the ground state $v_{1}$ satisfies

$$
L_{1}^{2} v_{1}=\frac{2(2 h(2,1)+1)}{3} L_{2} v_{1}
$$

The space $V_{2,1}^{s}$ is spanned by $v_{1}$ and $L_{1} v_{1}$, since $L_{1}^{2} v_{1}$ already belongs to $V_{1}^{a}$. In particular, the representation $V_{2,1}$ is quasirational, even if it is just degenerate and not minimal. Consider the fusion product with some $V_{r, s}$ with lowest weight state $v_{2}$. Since $\mathcal{A}_{0}$ reduces to $L_{0}$, we just have to calculate

$$
L_{0}\left(v_{1} \otimes v_{2}\right)=(h(2,1)+h(r, s))\left(v_{1} \otimes v_{2}\right)+z_{1}\left(L_{1} v_{1} \otimes v_{2}\right)
$$

and

$$
L_{0}\left(L_{1} v_{1} \otimes v_{1}\right)=(h(2,1)+h(r, s)+1)\left(L_{1} v_{1} \otimes v_{2}\right)+z_{1}\left(L_{1}^{2} v_{1} \otimes v_{2}\right) .
$$

The right hand side of the latter expression reduces to

$$
L_{0}\left(L_{1} v_{1} \otimes v_{2}\right) \equiv \frac{2 h(r, s)(2 h(2,1)+1)}{3} z_{1}^{-1}\left(v_{1} \otimes v_{2}\right)+\frac{3 h(r, s)-h(2,1)+1}{3}\left(L_{1} v_{1} \otimes v_{2}\right)
$$

Diagonalization immediately yields the eigenvalues $h(r+1, s)$ and $h(r-1, s)$ of $L_{0}$ on $V_{2,1}^{s} \otimes$ $V_{r, s}$, as expected. For minimal representations, one of the eigenvalues may be spurious. For degenerate representations, the quantum dimensions are integral and immediately calculable. For generic $c$, one reads of that the fusion ring of the degenerate representations 
is isomorphic to the tensor ring of $S U(2) \times S U(2)$. For $c=1$ one can put $p=q=s=1$. The fusion ring of the degenerate representations reduces to the fusion ring of $S U(2)$. Now let us consider the fusion of generic representations with ground states $v_{1}, v_{2}$ and lowest weight eigenvalues $h_{1}, h_{2}$ of $L_{0}$. Here, $V_{1}^{s}$ is given by the action of the polynomials of $L_{1}$ on $v_{1}$, without any relations, whereas $V_{2}^{l}$ is one dimensional. Thus the lowest weight space $V_{1}^{s} \otimes V_{2}^{l}$ is isomorphic to the ring of polynomials $p(x)$ in one variable. When one puts $x=h_{1}+h_{2}+z_{1} L_{1}$, the action of $L_{0}$ is just given by multiplication with $x$. This representation has infinite dimension, only its finite dimensional factor spaces induce representations in $\mathcal{V}$. Those are of course obtained by factoring out the ideal generated by some polynomial $f(x)$. The zeros of $f(x)$ yield the lowest weight eigenvalues of $L_{0}$. If no multiple zeros occur, one obtains a completely reducible representation. Since the zeros can be chosen arbitrarily, all possible values of the lowest weights are permitted in the fusion product, but only with multiplicity one. Multiple zeros yield reducible but indecomposable representations, whose $n$-point functions involve logarithms.

\section{Open questions}

Here is a list of the major questions posed above.

Are there lowest weight representations of some $W$-algebra which have finite quantum dimension but are not quasirational?

Are there conformal quantum field theories in two dimensions whose $W$-algebras are not quasirational?

Of similar difficulty may be the construction of universal algorithms for the following problems: Check if $W$-algebras or particular representations of some $W$-algebra are quasirational. Find the spurious representations of the zero mode algebra in the fusion product algorithm described above.

An easier problem should be the proof that standard construction procedures for $W$ algebras like orbifolding or GKO reductions always yield quasirational $W$-algebras when applied to such algebras.

Acknowledgement I thank W. Eholzer and R. Hübel for useful discussions and suggestions. 


\section{References}

[FF 88] B.L.Feigin and D.B.Fuchs, J.Geom.Phys. 5, 209,1988

[FFK 89] G.Felder, J.Fröhlich, G.Keller, CMP 124, 647, 1989

[FFK 90] G.Felder, J.Fröhlich, G.Keller, CMP 130, 1, 1990

[FRS 89] K.Fredenhagen, K.H.Rehren, B.Schroer, CMP 125, 201, 1989

[G 93] M.Gaberdiel, Fusion in Conformal field theory as the tensor product of the symmetry algebra, DAMTP preprint, 1993

[KL 93] D.Kazhdan and G.Lusztig: Tensor structures arising from affine Lie Algebras I,II, preprints, 1993

[MMS 88] S.D.Mathur, S.Mukhi, A.Sen, Phys.Lett. B213, 303, 1988

[V 88] E.Verlinde, Nucl.Phys. B300, 360, 1988 